\title{
THE RECOVERY OF SOME ABELIAN GROUPS FROM THEIR SOCLES
}

\author{
PAUL HILL ${ }^{1}$
}

\begin{abstract}
The first main result of this paper is that summable isotype subgroups inherit total projectivity. This means: $(*)$ if an isotype subgroup of a totally projective group has a free socle (viewed as a valuated vector space) then the socle completely determines the subgroup up to isomorphism. The next major result is that $(*)$ does not generalize to the case where the socle is that of a totally projective group of length exceeding $\Omega$, nor does $(*)$ generalize to the case where the socle is that of an $S$-group of length $\Omega$. Finally, it is shown that if, in addition to $H$ being isotype in a d.s.c. group $G$, it is also known that $K / H$ is divisible where $K$ is the closure of $H$ in $G$ relative to the $p^{\Omega}$-topology, then $(*)$ again prevails when the socle is that of an $S$-group.
\end{abstract}

1. Introduction and preliminaries. This paper is an expansion and revision of a previous manuscript entitled "Summable isotype subgroups inherit total projectivity".

As is well known, the structure of countable primary groups was determined by $\mathrm{H}$. Ulm [16]. In his honor, the Kaplansky-Mackey numbers [11, 12] are often called "Ulm invariants". We are referring to the numbers $\operatorname{dim}\left(p^{\alpha} G[p] / p^{\alpha+1} G[p]\right)$; the structure of a reduced, countable Abelian $p$-group $G$ is completely reflected through these cardinal numbers. G. Kolettis [13] extended this result to a direct sum of countable groups, and the author further extended it to the wider class of totally projective groups [4]. Moreover, R. Warfield [17] has shown that even a larger class of groups, which he called $S$-groups, are determined by the Kaplansky-Mackey numbers together with some additional numbers. These additional invariants of Warfield can also be obtained from the socle, viewed as a valuated vector space, in case the length of the group does not exceed $\Omega$ but not in general.

Following R. Nunke [14], we use the abbreviation "d.s.c." for "direct sum of countable (groups)". All groups herein are assumed to be Abelian, and we restrict our attention to the $p$-primary case. The smbol $h_{G}(x)$ is used to denote the height of $x$ in $G$. Thus $h_{G}(x)=\lambda$ if $x \in p^{\lambda} G$ but $x \notin p^{\lambda+1} G$. A subgroup $H$ of $G$ is isotype if $p^{\lambda} G \cap H=p^{\lambda} H$ for all $\lambda$.

Pure subgroups have for a long time been significant in the development of the theory of abelian groups; however, as the theory of $p$-groups began to focus more on groups of infinite and even uncountable length, the more general concept of

Received by the editors July 27, 1981 and, in revised form, April 7, 1982.

1980 Mathematics Subject Classification. Primary 20K25, 20K10, 20K27; Secondary 20K12, 20 K40.

Key words and phrases. Primary abelian group, isotype, summable, totally projective, separable subgroup, d.s.c., $S$-group, socle, valuated vector space.

${ }^{1}$ Sponsored by NSF Grant MCS 8102470. 
isotypeness often became more relevant than purity. Moreover, the interest in isotype subgroups, especially of a d.s.c., totally projective, or of an $S$-group, is enhanced for at least two further reasons which are related to the current interest in valuated groups and vector spaces. First, an isotype subgroup is simply a subgroup when the group is endowed with its natural valuation of height, $|x|=h_{G}(x)$. Secondly, since the structure of a d.s.c., totally projective, or of an $S$-group of length $\Omega$ is completely determined by its socle (the underlying valuated vector space), it is natural to inquire whether or not an isotype (= valuated) subgroup of one of these groups is determined by its socle, too, without knowing a priori that the subgroup belongs to one of structural classes listed. For example, consider the following.

Problem. Suppose that $H$ is an isotype subgroup of a totally projective group $G$. Does the valuated vector space $H[p]$ reveal whether or not $H$, itself, is totally projective?

Observe that if the revelation of the socle $H[p]$ should be that $H$ is totally projective, then $H$ can be entirely recovered from $H[p]$ because the socle contains the Ulm invariants. We demonstrate in this paper that the answer to the preceding problem depends on the length of $G$. Let us mention what is already known about the problem. First, consider the case where the length of $G$ does not exceed $\Omega$; in other words, consider the case that $G$ is a d.s.c. If the length of $G$ is countable, then by only a perfunctory examination of $H[p]$ we conclude that $H$ is a d.s.c. because the outcome is already certain $[5,9]$. However, a d.s.c. of countable length is, in a sense, not a typical d.s.c. The interesting case where the length is $\Omega$ has been an open question since it was posed in [5] in the following form. If the isotype subgroup $H$ of a d.s.c. is summable, must $H$ be a d.s.c.? For length $\Omega$, this question is equivalent to the Problem because a reduced $p$-group $G$ is summable if and only if its socle $S=G[p]$ is a free valuated vector space, and a d.s.c. must be summable [2].

It is well documented that a summable $C_{\Omega}$-group need not be a d.s.c. $[6,1]$. Recall that $H$ is a $C_{\Omega}$-group provided that $H / p^{\alpha} H$, if not $H$ itself, is a d.s.c. for each countable $\alpha$. An isotype subgroup of a d.s.c. must be a $C_{\Omega}$-group, but the converse is false and the Problem remained unsolved with this result. However, an affirmative solution is given in the next section for length $\Omega$ using some recent concepts and results from valuated vector spaces.

2. Summable isotype subgroups inherit total projectivity. A concept that will be useful is that of a separable subgroup. This concept and terminology is taken from valuated vector spaces [7]; it is not to be confused with the notion of separability found in [2].

Definition 1. A subgroup $H$ of $G$ is separable if, for each $g \in G$, there exists a countable sequence of elements $x_{n} \in H$ such that

$$
\sup \left\{h_{G}(g+x): x \in H\right\}=\sup \left\{h_{G}\left(g+x_{n}\right)\right\}_{n<\omega} .
$$

Another relatively new concept, which was first introduced by the author for valuated vector spaces in [7], is the following.

Definition 2. Let $A$ and $B$ be subgroups of the $p$-group $G$. We say that $A$ and $B$ are compatible, and write $A \| B$, if for each pair $(a, b) \in A \times B$ there exists $c$ in $A \cap B$ such that $h_{G}(a+c) \geqslant h_{G}(a+b)$. 
The next result is essential. Although it appears in [8], we include a short proof.

LEMMA 1. Let $H$ be a separable subgroup of $G$. If $A$ is any infinite subgroup of $G$, there is a subgroup $B$ of $G$ having the same cardinality as $A$ and containing $A$ such that $B \| H$.

Proof. Set $B_{0}=A$ and assume that $B_{i}$ has been chosen. For each $b \in B_{i}$ select a sequence of elements $x_{n}^{b}$ in $H$ so that

$$
\sup \left\{h_{G}\left(b+x_{n}^{b}\right)\right\}_{n<\omega}=\sup \left\{h_{G}(b+x): x \in H\right\} ;
$$

this is possible since $H$ is separable in $G$. Define

$$
B_{i+1}=\left\langle B_{i}, x_{n}^{b}\right\rangle_{b \in B_{i}, n<\omega},
$$

and let $B=\cup_{i<\omega} B_{i}$. Observe that $B$ has the same cardinality as $A=B_{0}$. Suppose that $h_{G}(b+x)=\lambda$ where $b \in B$ and $x \in H$. Since $b \in B_{i}$ for some $i$, there exists $c=x_{n}^{b}$ in $B \cap H$ such that $h_{G}(b+c) \geqslant \lambda$. This shows that $B \| H$, and the lemma is proved.

The next lemma is well known, and is included here only for reference. For example, it appears as an exercise in [2].

LEMMA 2. Let $G$ be a reduced p-group of length not exceeding $\Omega$. Any infinite subgroup of $G$ is contained in an isotype subgroup of the same cardinality.

The following observation is valuable.

LEMMA 3. Let $H$ be a isotype subgroup of $G$. If $H$ is summable, then $H$ is separable in $G$.

Proof. Suppose that $H$ is isotype and summable but is not separable in $G$. Since $H$ is summable, $p^{\Omega} H=0$ by Theorem 84.3 in [2]. Thus we may assume that $p^{\Omega} G=0$, for $H$ remains isotype and nonseparable in $G / p^{\Omega} G$. Assume that

$$
\sup \left\{h_{G}(g+x): x \in H\right\}=\Omega
$$

with $g \notin H$. Then such an element $g$ must exist with $p g \in H$. Since $H$ is isotype we may assume that $p g=0$. Furthermore, for each $\alpha<\Omega$, there exists $h_{\alpha} \in H$ such that $h_{G}\left(g+h_{\alpha}\right)>\alpha$, and we may, in fact, choose $h_{\alpha}$ so that $p h_{\alpha}=0$. We have now obtained a contradiction because the free valuated vector space $H[p]$ must be separable in $G[p]$ according to [7, Theorem 1].

THEOREM 1. Suppose that $H$ is an isotype subgroup of a d.s.c. Then $H$ is a d.s.c. if its socle permits, being interpreted if $H$ is summable or its socle is free.

Proof. Let $G$ be the containing d.s.c., and write $G=\Sigma_{i \in I} C_{i}$ where $C_{i}$ is countable for each $i$. If $J \subseteq I$, let $G(J)=\sum_{j \in J} C_{j}$. For our purposes, the set $J$ will depend on an ordinal $\alpha$ and will appear as $J(\alpha)$. In this case, we write $G(\alpha)$ instead of $G(J(\alpha))$. Observe that $G(J)$ is also a d.s.c. Since $H$ is summable, $H[p]$ has a basis as a valuated vector space. Thus we write

$$
H[p]=\sum_{t \in T}\left\langle b_{t}\right\rangle,
$$


where $h_{H}\left(\sum n_{t} b_{t}\right)=\min \left\{h_{H}\left(n_{t} b_{t}\right)\right\}$. And we set $H[p]_{S}=\Sigma_{s \in S}\left\langle b_{s}\right\rangle$ for any subset $S$ of $T$.

The proof of the theorem is by induction on the cardinality of $H$. If $H$ is countable, there is nothing to prove. Therefore, we assume that $|H|=m>\boldsymbol{\aleph}_{0}$. The proof also will be based on the existence of a chain

(c) $0=J(0) \subseteq J(1) \subseteq \cdots \subseteq J(\alpha) \subseteq \ldots, \alpha<\tau$, of subsets $J(\alpha)$ of $I$ satisfying the following conditions:

(1) $G(\alpha) \cap H$ is isotype (in $H$ ).

(2) $G(\alpha) \cap H[p]=H[p]_{S(\alpha)}$ for some subset $S(\alpha)$ of $T$.

(3) $G(\alpha) \| H$.

(4) $J(\beta)=\cup_{\alpha<\beta} J(\alpha)$ when $\beta$ is a limit. $\left(G(\beta)=\cup_{\alpha<\beta} G(\alpha)\right.$.)

(5) $H \subseteq \cup_{\alpha<\tau} G(\alpha)$.

(6) $|J(\alpha)|<m .(|G(\alpha)|<m$.)

To obtain both (5) and (6), some efficiency is needed in the development of the chain of subsets $J(\alpha)$. More precisely, we need the length $\tau$ of the chain to be as small as possible. Thus we preselect $\tau$ to be the smallest ordinal such that $m=$ $\sup \left\{m_{\alpha}\right\}_{\alpha<\tau}$ where $m_{\alpha}$ is a smaller cardinal than $m$. To ensure (5) and (6), we let $H=\cup_{\alpha<\tau} A_{\alpha}$ where $\left|A_{\alpha}\right|=m_{\alpha}<m$. Then, for each $\alpha<\tau$, we always choose $J(\alpha+1)$ so that $A_{\alpha} \subseteq G(\alpha+1)$. Since each of the properties (1)-(3) is inductive, there is no problem in the construction of the desired chain of subsets $J(\alpha)$ when a limit ordinal is approached, for we simply honor (4) and let $J(\beta)=\cup_{\alpha<\beta} J(\alpha)$. Observe that $|J(\beta)|<m$, for otherwise condition (5) is already achieved at $\tau=\beta$ and the chain terminates prior to $J(\beta)$. For an isolated ordinal $\alpha+1$, we know that $|J(\alpha)|<m$ and, of course, $\left|A_{\alpha}\right|<m$. Applications of Lemmas 1 and 2 with alternate consideration of the individual properties (through the "back-and-forth" procedure) yield a set $J(\alpha+1)$ satisfying conditions (1)-(3) with $|J(\alpha+1)|<m$ and $A_{\alpha} \subseteq$ $G(\alpha+1)$. We now consider the argument complete as far as the existence of the chain (c) satisfying (1)-(6) is concerned.

For each $\alpha<\tau$, set $H(\alpha)=G(\alpha) \cap H$. It is a consequence of the conditions on $G(\alpha)$ that $H(\alpha)$ is a balanced subgroup of $H(\alpha+1)$. Immediately, $H(\alpha)$ is isotype in $H(\alpha+1)$; in fact, $H(\alpha)$ is isotype in $G$ by (1). Thus we need only show that $H(\alpha)$ is nice in $H(\alpha+1)$ in order to prove that it is a balanced subgroup. Suppose that $x \in H(\alpha+1)$, and let

$$
h_{H(\alpha+1) / H(\alpha)}(x+H(\alpha))=\lambda .
$$

It follows trivially that

$$
h_{G / G(\alpha)}(x+G(\alpha)) \geqslant \lambda .
$$

Since $G(\alpha)$ is a summand of $G$, there exists $g \in G(\alpha)$ such that $h_{G}(x-g) \geqslant \lambda$; namely, the projection of $x$ upon $G(\alpha)$. Condition (3) implies that $h_{G}(x+c) \geqslant \lambda$ for some $c$ in $H(\alpha)$. Thus $h_{H(\alpha+1)}(x+c) \geqslant \lambda$ because $H(\alpha+1)$ is isotype in $G$, and $H(\alpha)$ is nice in $H(\alpha+1)$.

Since $H(0)=0$ and $H(\beta)=\cup_{\alpha<\beta} H(\alpha)$ when $\beta$ is a limit, it follows that

$$
H=\sum_{\alpha<\tau} D_{\alpha}
$$


provided $H(\alpha+1)=H(\alpha) \oplus D_{\alpha}$. Thus the proof of the theorem will be finished if we can show that $H(\alpha+1) / H(\alpha)$ is a d.s.c. because a d.s.c. is a balanced projective [2, Theorem 81.9] and $H(\alpha)$ is balanced in $H(\alpha+1)$.

We claim that $H(\alpha+1) / H(\alpha)$ is summable; equivalently, $(H(\alpha+1) / H(\alpha))[p]$ is a free valuated vector space. Since $H(\alpha)$ is isotype,

$$
(H(\alpha+1) / H(\alpha))[p]=\langle H(\alpha+1)[p], H(\alpha)\rangle / H(\alpha) .
$$

Hence condition (2) yields an isomorphism

$$
(H(\alpha+1) / H(\alpha))[p] \cong H[p]_{S(\alpha+1)-S(\alpha)} \quad\left(=\sum\left\langle b_{s}\right\rangle\right)
$$

that preserves heights. Thus $(H(\alpha+1) / H(\alpha))[p]$ is a free valuated vector space, and $H(\alpha+1) / H(\alpha)$ is summable.

Now consider the isomorphism

$$
H(\alpha+1) / H(\alpha) \cong\langle H(\alpha+1), G(\alpha)\rangle / G(\alpha) .
$$

We show that $\langle H(\alpha+1), G(\alpha)\rangle / G(\alpha)$ is isotype in $G / G(\alpha)$. Let $x \in H(\alpha+1)$ and suppose that

$$
h_{G / G(\alpha)}(x+G(\alpha))=\lambda \text {. }
$$

There exist $g \in G(\alpha)$ such that $h_{G}(x+g)=\lambda$. Condition (3) implies that $h_{G}(x+c) \geqslant \lambda$ for some $c \in H(\alpha)=H \cap G(\alpha)$. Since $H(\alpha+1)$ is isotype, $h_{H(\alpha+1)}(x+c) \geqslant \lambda$. Therefore, $x+G(\alpha)$ has height at least $\lambda$ in $\langle H(\alpha+1)$, $G(\alpha)\rangle / G(\alpha)$, so it is clear that $\langle H(\alpha+1), G(\alpha)\rangle / G(\alpha)$ is isotype in $G / G(\alpha)$. It is also summable because it is isomorphic to $H(\alpha+1) / H(\alpha)$. Moreover, the cardinality of $H(\alpha+1) / H(\alpha)$ is less than $m$. Thus the induction hypothesis is applicable. Whence $H(\alpha+1) / H(\alpha)$ is a d.s.c., and the theorem is proved.

We have stated the theorem in its basic form, but it has the following more general versions as a consequence of the transitive property of isotype subgroups.

COROLlary 1. A summable isotype subgroup inherits total projectivity.

Proof. Let $H$ be a summable isotype subgroup of a totally projective group $G$. Since $H$ is summable, its length does not exceed $\Omega$ [2, Theorem 84.3]. Thus $H$ appears as an isotype subgroup of $G / p^{\Omega} G$, which is a d.s.c. Theorem 1 finishes the proof.

COROllary 2. Suppose that $G$ can be imbedded as an isotype subgroup of a totally projective group, and let $H$ be an isotype subgroup of $G$. If $H$ is summable then $H$ is a d.s.c.

Proof. The property of being isotype is transitive. Then $H$ is totally projective whether $G$ is or not.

An important feature of $S$-groups is that they appear as isotype subgroups of totally projective groups. For a complete characterization of $S$-groups, see [17] or [10]. The preceding facts yield

COROLlary 3. A summable isotype subgroup of an S-group is a d.s.c. 
3. The insufficiency of a subfree socle in determining the structure of an isotype subgroup of a totally projective group. If a group has the socle of some totally projective group, call the group subsummable; Fuchs called such a socle subfree in [3]. It might appear reasonable to conjecture that if $H$ is an isotype subgroup of a totally projective group, then $H$ is totally projective if its socle permits, that is, if $H$ is subsummable. However, the following example shows that this is not the case.

Let $G$ be a totally projective group of length not less than $\Omega+1$ and let $H$ be a $p^{\Omega}$-high subgroup of $G ; H$ is maximal with respect to $H \cap p^{\Omega} G=0$. Since $H$ is isotype in $G$, we know that $H \oplus \sum G$ is isotype in $G \oplus \Sigma G=\Sigma G$, where $\Sigma G$ represents an infinite number of copies of $G$. Notice that the valuated vector space $G[p]$ decomposes as $G[p]=H[p] \oplus p^{\Omega} G[p]$. Therefore, $H \oplus \Sigma G$ and $G \oplus \Sigma G$, as well as $\Sigma G$, have isomorphic socles. This means, in particular, that $H \oplus \sum G$ is subsummable; however, the group is not totally projective since $H$ is not totally projective. The conclusion drawn from this example is stated in the next proposition.

PROPOSITION. The structure of an isotype subgroup of a totally projective group of length greater than $\Omega$ cannot, in general, be entirely recovered from its socle, even if that socle is subfree.

Remark. The Proposition means that one cannot determine from its socle alone whether or not an isotype subgroup of a totally projective group is itself, totally projective. Hence our Problem has a negative solution if the length of $G$ is beyond $\Omega$.

4. $S$-groups. Earlier we mentioned that an important feature of $S$-groups is that they are isotype subgroups of totally projective groups. Thus we are interested in the identification of an $S$-group through its socle. The preceding Proposition restricts our consideration to $S$-groups of length not exceeding $\Omega$. The following characterization of such an $S$-group is useful: an $S$-group of length $\Omega$ is an isotype subgroup of a d.s.c. that is dense in the $p^{\Omega}$-topology; an $S$-group of shorter length is a d.s.c. Before presenting our main positive result on $S$-groups, we establish the following.

THEOREM 2. There exists an isotype subgroup of a d.s.c. that has the socle of an $S$-group but is not an $S$-group.

Proof. Let $T$ be the standard totally projective group of length $\Omega+1$; all the Ulm invariants, $f_{\alpha}(T)$, for countable $\alpha$ are $\aleph_{1}$ and $f_{\Omega}(T)=1$. Let $p^{\Omega} T=\langle c\rangle$. We claim that there is an isotype subgroup $H$ of a d.s.c. group $G$ such that $G / H=T$. In fact, the detailed construction of such an example can be found in [8]. Briefly and perhaps more elegantly the idea is as follows. Let $G$ be the external sum of all countable subgroups $A$ of $T$ with the property that $\left\langle A, p^{\Omega} T\right\rangle / p^{\Omega} T$ is isotype in $T / p^{\Omega} T$. For each countable $\alpha$, there is such a subgroup $A_{\alpha}$ with the additional property that $p^{\alpha} A_{\alpha}$ contains $c$, the generator of $p^{\Omega} T$. Hence $A_{\alpha}$ can be chosen "arbitrarily close" to being isotype in $T$, but not quite since $A_{\alpha}$ contains an element of uncountable height in $T$. Now, the desired result is obtained if we let $H$ be the kernel of the natural map of $G$ onto $T$. We may assume without loss of generality that each nonzero Ulm invariant for both $G$ and $H$ is $\boldsymbol{N}_{1}$ because a superfluous d.s.c. summand can be deleted from or added to $G$. For simplicity of notation, let $G / H=T$. Now, $H$ is not an $S$-group since the closure of $H$ in the d.s.c. group $G$ 
relative to the $p^{\Omega}$-topology is finite but not zero. Alternately, it can be shown directly using cotorsion hulls that the Warfield invariant $k(\Omega, H)$ is zero.

To finish the proof of the theorem we need to show that $H$ has the socle of an $S$-group. Let $\bar{S}$ denote the closure of $H[p]$ in $G[p]$ with respect to the $p^{\Omega}$-topology. If we choose $g \in G[p]$ so that $\langle g+H\rangle=p^{\Omega} T$, then we know that $\bar{S}=\langle H[p], g\rangle$. In order to show that $H[p]$ is the socle of an $S$-group, it suffices to prove that $\bar{S}=\langle H[p], g\rangle$ is free. For in this case, there is a d.s.c. with socle $\bar{S}$ and with an isotype dense subgroup (hence an $S$-group) whose socle is $H[p]$. To prove that $\bar{S}$ is free (as a valuated vector space) we look at $\bar{S}$ in $G[p]$. Since $G$ is a d.s.c., we know that $G[p]$ is free. Furthermore, $G[p] / \bar{S}$ is the quotient of a free space by a free space ( $Q F F$ space) according to [7, Proposition 2] since the cardinality of $G[p]$ is only $\boldsymbol{\aleph}_{1}$. The relation

$$
\sup \left\{h_{G}(g+x): x \in \bar{S}\right\}<\Omega
$$

for each $g \in G[p] \backslash \bar{S}$ demonstrates that $\bar{S}$ is separable in $G[p]$. Therefore, $\bar{S}$ is free by [7, Theorem 6], and the proof of the theorem is finished.

Now let $G$ denote an arbitrary d.s.c. If an isotype subgroup $H$ of $G$ is, in fact, an $S$-group, then $K / H$ must be divisible where $K$ is the closure of $H$ in $G$ relative to the $p^{\Omega}$-topology. This property motivates the following definition.

Definition 3. An isotype subgroup $H$ of a d.s.c. is said to be uniform if $K / H$ is divisible, where $K$ is the closure of $H$ in $G$ relative to the $p^{\Omega}$-topology.

Although an $S$-group of length $\Omega$ must appear as a uniform isotype subgroup of a d.s.c., the converse is not true. For example, any balanced subgroup of a d.s.c. is trivially a uniform isotype subgroup, but there are balanced subgroups of a d.s.c. that are neither d.s.c. groups not $S$-groups [15]. However, the next theorem shows that one can determine whether a uniform isotype subgroup of a d.s.c. is an $S$-group by looking at its socle. In the affirmative case, the whole group can then be recovered from its socle by Warfield's theorem [17].

THEOREM 3. Let $H$ be a uniform isotype subgroup of a d.s.c. group $G$. Then $H$ is an $S$-group if its socle permits, that is, if $H$ has the socle of an $S$-group.

Proof. Consider the closure $K$ of $H$ in $G$ relative to the $p^{\Omega}$-topology,

$$
K=\left\{g \in G: \sup \left\{h_{G}(g+x): x \in H\right\}=\Omega\right\} .
$$

Since $H[p]$ is the socle of an $S$-group, we know that $K[p]$ is free. Since $H$ is uniform, $K / H$ is divisible. From the divisibility of $K / H$ it is easy to show by induction that $p^{\alpha} G \cap K \subseteq p^{\alpha} K$ for all $\alpha$. Thus $K$ is isotype in $G$. The crux of the proof is that $K$ is therefore a d.s.c. according to Theorem 1. It is now routine to observe that $H$ is isotype and dense in $K$. Thus $H$ is an $S$-group.

\section{REFERENCES}

1. D. Cutler, Another summable $C_{\Omega^{-}}$group, Proc. Amer. Math. Soc. 26 (1970), 43-44.

2. L. Fuchs, Infinite abelian groups, Vol. II, Academic Press, New York, 1973.

3. __ Subfree valued vector spaces, Lecture Notes in Math., vol. 616, Springer-Verlag, Berlin and New York, 1977, pp. 158-167.

4. P. Hill, On the classification of abelian groups, photocopied manuscript, Houston, 1967.

5. __ Isotype subgroups of direct sums of countable groups, Illinois J. Math. 13 (1969), 281-290. 
6. __ A summable $C_{\Omega^{-}}$group, Proc. Amer. Math. Soc. 23 (1969), 428-430.

7. $ـ$ Criteria for freeness in abelian groups and valuated vector spaces, Lecture Notes in Math., vol. 616, Springer-Verlag, Berlin and New York, 1977, pp. 145-157.

8. _ Isotype subgroups of totally projective groups, Lecture Notes in Math., Springer-Verlag, Berlin and New York (to appear).

9. P. Hill and C. Megibben, On direct sums of countable groups and generalizations, Studies on Abelian Groups, Dunod, Paris, 1968, pp. 183-206.

10. R. Hunter and E. Walker, $S$-groups revisited, Proc. Amer. Math. Soc. 82 (1981), 13-18.

11. I. Kaplansky, Infinite abelian groups, Univ. of Michigan Press, Ann Arbor, Michigan, 1969.

12. I. Kaplansky and .G. Mackey, A generalization of Ulm's theorem, Summa Brasil. Math. 2 (1951), 195-202.

13. G. Kolettis, Direct sums of countable groups, Duke Math. J. 27 (1960), 111-125.

14. R. Nunke, Homology and direct sums of countable abelian groups, Math. Z. 101 (1967), 182-212.

15. F. Richman and E. Walker, Valuated groups, J. Algebra 56 (1979), 145-167.

16. H. Ulm, Zur Theorie der abzahlbar-unendlichen abelischen Gruppen, Math. Ann. 107 (1933), 774-803.

17. R. Warfield, Jr., A classification theorem for abelian p-groups, Trans. Amer. Math. Soc. 210 (1975), 149-168.

Department of Mathematics, AUburn University, Auburn University, Alabama 36849 